\title{
Covariance Applications in Criticality Safety, Light Water Reactor Analysis, and Spent Fuel Characterization
}

\author{
M.L. Williams, ${ }^{1, *}$ D. Wiarda, ${ }^{1}$ G. Ilas, ${ }^{1}$ W.J. Marshall,${ }^{1}$ and B.T. Rearden ${ }^{1}$ \\ ${ }^{1}$ Reactor and Nuclear Systems Division, Oak Ridge National Laboratory, Oak Ridge, TN 37831, USA
}

(Received 15 June 2014; accepted 21 July 2014)

\begin{abstract}
A new covariance data library based on ENDF/B-VII.1 was recently processed for the SCALE nuclear analysis code system. The multigroup covariance data are discussed here, along with testing and application results for critical benchmark experiments. The cross section covariance library, along with covariances for fission product yields and decay data, is used to compute uncertainties in the decay heat produced by a burned reactor fuel assembly.
\end{abstract}

\section{INTRODUCTION}

The SCALE computational system [1] developed by Oak Ridge National Laboratory is widely used for diverse types of nuclear applications, including reactor lattice physics, criticality safety, radiation shielding, and spent fuel characterization. The latest version, SCALE6.2 , is scheduled for release in 2014. A notable feature of SCALE is that it includes modules and data for performing various types of sensitivity and uncertainty (SU) analysis. A statistical sampling module called Sampler 2] has been developed for SCALE-6.2 to complement SU techniques based on generalized perturbation theory used by the TSUNAMI (Tools for Sensitivity and Uncertainty Analysis Methodology Implementation) modules [3]. The new capability provides robust methods for uncertainty quantification of virtually any multigroup (MG) application addressed by SCALE, as long as uncertainty information is available for the various types of input nuclear data used in the calculations.

The AMPX code system [4 was recently used to process new continuous energy (CE) and MG nuclear data libraries from Evaluated Nuclear Data File B, Version VII.1 (ENDF/B-VII.1) [5] data for SCALE-6.2. In addition, new and revised covariance libraries for reaction cross sections, fission product yields, and decay data will be included in SCALE-6.2. This paper describes the covariance libraries in SCALE-6.2 and how they were gen-

\footnotetext{
* Corresponding author: williamsml@ornl.gov

This manuscript has been authored by UT-Battelle LLC under Contract No. DE-AC05-00OR22725 with the US Department of Energy. The US government retains and the publisher, by accepting the article for publication, acknowledges that the US government retains a nonexclusive, paid-up, irrevocable, worldwide license to publish or reproduce the published form of this manuscript, or allow others to do so, for US government purposes.
}

erated. In addition, several applications of the new covariance data are presented.

\section{COVARIANCE DATA FOR SCALE-6.2}

\section{A. Modifications and Extensions}

The previously released version 6.1 of SCALE includes a 44 MG covariance library based on several sources of uncertainty data, including ENDF/VII.0 and approximate "low-fidelity" representations 6]. For SCALE-6.2, the PUFF module of AMPX was used to process new MG covariance data from ENDF/B-VII.1, which contains many more evaluated covariances than available in ENDF/B VII.0. The processed ENDF/B-VII.1 covariances for isotopes important in criticality safety and burnup credit analysis were compared to values in the earlier SCALE covariance library. The ENDF/B-VII.1 covariance data appear reasonable and generally agree quite well with the earlier SCALE uncertainty values for many isotopesnotably for fission product nuclides that were based on the low-fidelity representation in SCALE-6.1. However, two issues were found in the major actinides. The standard deviation in ${ }^{239} \mathrm{Pu}$ nubar is zero in the range below $0.01 \mathrm{eV}$. For the SCALE-6.2 library this uncertainty will be modified by extending the value of $0.15 \%$ from 0.01 to $0.0 \mathrm{eV}$. A possible problem was also observed in the ${ }^{235} \mathrm{U}$ thermal nubar uncertainty, which is shown in Fig. 1. ENDF/B-VII has a standard deviation of about $0.7 \%$, which is twice the uncertainty value in SCALE and in other evaluations such as the Japanese Evaluated Nuclear Data Library. The ENDF/B-VII.1 ${ }^{235} \mathrm{U}$ covariance will be modified for SCALE- 6.2 by reducing the standard deviation in thermal nubar to the SCALE-6.1 value of $0.3 \%$ 77. Finally, the ENDF/B-VII uncertainty in the hydrogen thermal cross section has been reduced from $2.5 \%$ to 
$0.2 \%$. ENDF/B-VII.1 also includes new covariance data for fission neutron spectra of 65 actinides, and these data have been processed with PUFF for the SCALE-6.2 library. Fission spectra covariances in earlier versions of SCALE were based on the Watt spectrum.

In addition to covariances for reaction cross section data, SCALE-6.2 will also include covariances for fission product yields, decay constants, and branching fractions, which are required for reactor burnup calculations and analysis of used nuclear fuel. Uncertainties for these data were obtained from ENDF/B-VII.1 decay files. Covariances for decay constants are assumed to be uncorrelated, while branching fractions are correlated by the requirement that they must sum to unity. Covariance data for fission product yields are generated using the method developed by Pigni et al. in which correlations in the fission product yield uncertainties are obtained by combining ENDF/B-VII.1 uncertainties for independent and cumulative yields [8].

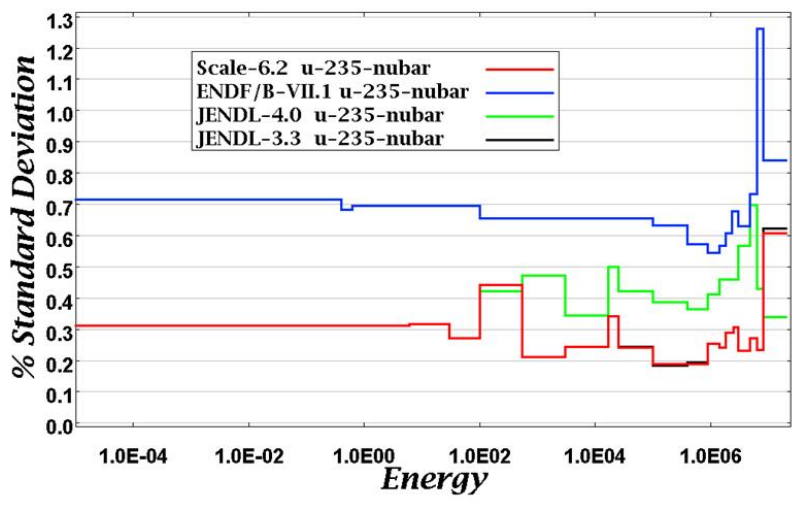

FIG. 1. (Color online) Standard deviation in ${ }^{235} \mathrm{U}$ nubar.

\section{B. Analysis for Benchmark Critical Experiments}

The ENDF/B-VII.1 cross section and covariance libraries have been tested using SCALE-6.2 calculations for more than 300 critical benchmark experiments described in the International Handbook of Evaluated Criticality Safety Benchmark Experiments, which provides experimental values for multiplication factors, along with experimental uncertainties, for several categories of critical systems [9]. In this paper we focus on the following three categories of uranium-fueled, thermal-spectra systems: (a) high-enriched, thermal solution systems (HST), (b) low-enriched, thermal solution systems (LST), and (c) low-enriched, thermal lattice systems (LCT).

Multiplication factors for the critical experiments were calculated with the KENO Monte Carlo code using both $\mathrm{CE}$ and MG ENDF/B-VII.1 nuclear data libraries. Uncertainty analysis was performed with the TSUNAMI3D module, which uses MG Monte Carlo calculations to obtain forward and adjoint fluxes from which eigen- value sensitivity coefficients are computed using perturbation theory 3 . The sensitivity coefficients are combined with MG covariance data to obtain uncertainties in the computed system eigenvalues due to nuclear data uncertainties. Because Monte Carlo models have few geometric approximations and the statistical uncertainties $(<10 \mathrm{pcm})$ are much smaller than the quoted experiment uncertainty, the differences between the calculated and experiment multiplication factors are due mainly to uncertainties in the basic nuclear data and processing methods.

Figures 2-4 are plots of the ratios of calculated to experimental $(\mathrm{C} / \mathrm{E})$ multiplication factors for the benchmark cases, along with uncertainty bands corresponding to plus or minus one standard deviation for the experimental measurements. The agreement between $\mathrm{CE}$ and MG results is generally consistent, which provides confidence that the computed uncertainty values based on MG methods will be reasonable. The plots also include one-sigma uncertainty bands calculated with nuclear data covariances from the original ENDF/B-VII.1 evaluations as well as the modified ENDF/B-VII.1 data in SCALE6.2 .

The $\mathrm{C} / \mathrm{E}$ values computed with $\mathrm{MG}$ and $\mathrm{CE}$ methods lie within the experimental uncertainty for most benchmarks. For these uranium-fueled criticals, the calculated uncertainties using unaltered ENDF/B-VII.1 covariances are significantly larger than values obtained with SCALE6.2 covariances, due mainly to the reduced standard deviation for ${ }^{235} \mathrm{U}$ thermal nubar.

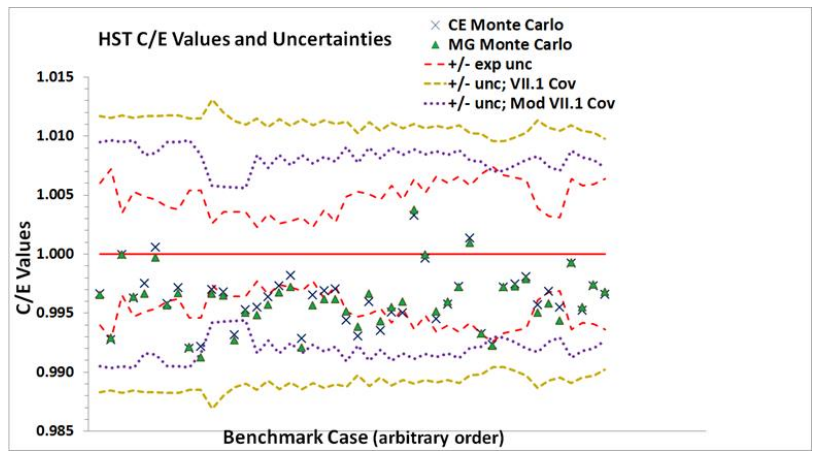

FIG. 2. (Color online) C/E for HEU thermal solutions.

It can also be seen that the variation in the calculated $\mathrm{C} / \mathrm{E}$ values for these benchmark experiments is significantly less than the predicted uncertainties calculated with ENDF/B-VII.1 differential covariance data, and to a lesser degree, with the SCALE-6.2 covariances. A common procedure in criticality safety validation is to determine the uncertainty for a given computational method and nuclear library by considering results of calculations for benchmark experiments similar to the application of interest. The average $\mathrm{C} / \mathrm{E}$ value is used to obtain the computation/data bias, while the computed sample standard deviation of $\mathrm{C} / \mathrm{E}$ results provides an indication of the expected uncertainty in a calculated application sys- 


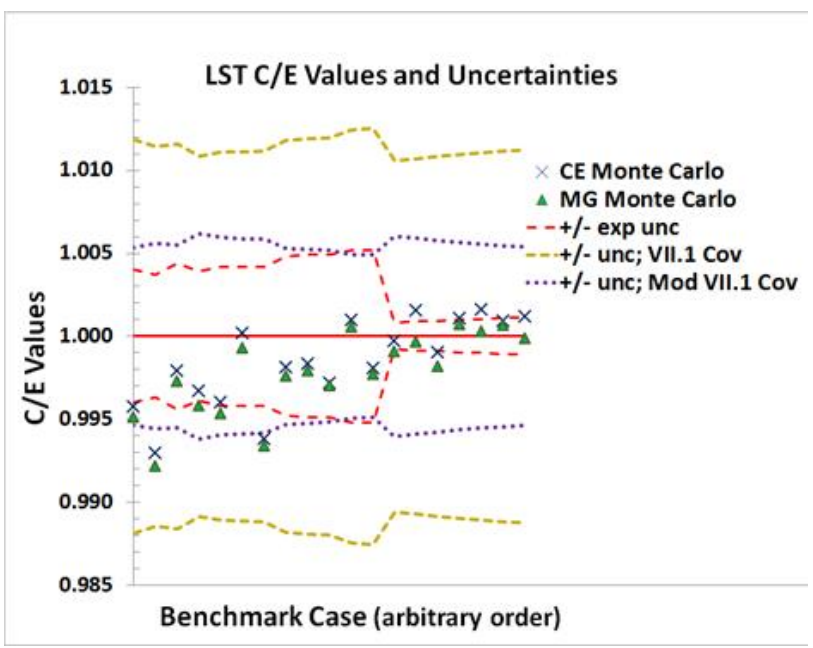

FIG. 3. (Color online) C/E for LEU thermal solutions.

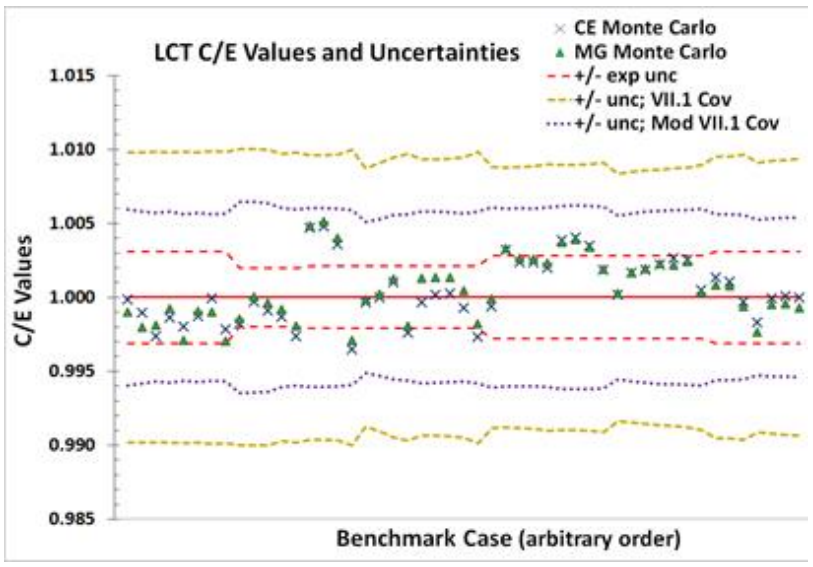

FIG. 4. (Color online) C/E for LEU lattices.

tem for which no experimental measurement is available. An alternative approach based on uncertainty analysis is to compute a priori estimates for the calculated uncertainties in the benchmark experiments, as well as the applications from the differential nuclear data uncertainties.

Table I compares, for each category, the relative sample standard deviations computed from the $\mathrm{C} / \mathrm{E}$ distributions versus the average value of the a priori standard deviation predicted by $\mathrm{SU}$ calculations using the unaltered ENDF/B-VII.1 covariances and the SCALE-6.5 modified version VII.1 covariances. The a priori standard deviations based on nuclear data uncertainties are significantly greater than indicated by the distribution of $\mathrm{C} / \mathrm{E}$ results for these benchmark experiments. These results cast some doubt on whether uncertainties computed using the current ENDF/B-VII.1 nuclear data covariances are predictive for eigenvalue uncertainties in light-watermoderated critical systems and, by implication, for power reactors. However, it is well known that prior uncertainties may be reduced by consolidating the calculated and the measured integral benchmark experiment results using generalized linear least-squares (GLLS) adjustment. After GLLS is performed, the initial a priori uncertainties will be reduced due to incorporation of additional information provided by the integral measurements. The results presented here suggest that it may be essential to apply GLLS methods to obtain realistic values for the predicted uncertainties in calculated critical eigenvalues, using differential covariance data in the current state.

\section{UNCERTAINTY ANALYSIS FOR USED NUCLEAR FUEL CHARACTERIZATION}

One of the primary challenges facing the nuclear power industry today is transportation and storage of used nuclear fuel (UNF). The US government requires a realistic assessment of UNF subcriticality margins, isotopic compositions, dose levels, and decay heats over a long time period spanning onsite storage, transportation in shipping casks, and ultimate disposal at a repository - as well as potential reprocessing operations. The reactivity reduction achieved by burnup credit for several specified fission products is also of interest. Reactor burnup calculations consisting of coupled neutron transport and isotopic depletion calculations are necessary to predict the properties of UNF. Uncertainties in the calculated radiological characteristics of UNF, which may impact the safety margins established by regulatory bodies, depend on neutron cross section covariances, uncertainties in fission product yields, decay constants, and decay energy.

The TRITON depletion sequence [10] in SCALE is used to perform burnup calculations for reactor lattices to determine reaction rates, activities, isotopic concentrations, etc. as a function of time. Reference 11 describes a study with the Sampler module to assess the uncertainties in burnup calculations by randomly perturbing input values for MG cross sections according to the SCALE-6.1 covariance information. A similar study to compute uncertainties in the time-dependent decay heat generation in UNF was recently completed using the SCALE-6.2 reaction covariance library based on ENDF/B-VII.1. Figure 5 illustrates the uncertainty in calculated decay heat for cooling times up to 300 years. After 300 years of cooling, the total decay heat decreases four orders of magnitude compared to the discharge time (i.e., from about 1 MW/MTU to about $\left.10^{-4} \mathrm{MW} / \mathrm{MTU}\right)$. The ${ }^{241} \mathrm{Am}$ concentration in spent fuel increases with increasing cooling time due to the $\beta$ decay of ${ }^{241} \mathrm{Pu}$ with a 14.4 -year halflife. For cooling times greater than 100 years, the uncertainty in the total decay heat is less than $2 \%$, which is attributable mainly to the ${ }^{241} \mathrm{Pu}$ concentration uncertainty as its daughter ${ }^{241} \mathrm{Am}$ is the main contributor to the decay heat for this cooling time range. 
TABLE I. Predicted standard deviations in $\mathrm{C} / \mathrm{E}$ values.

\begin{tabular}{c|c|c|c|c|c|c}
\hline \hline \multirow{2}{*}{ Case } & \multicolumn{2}{|c|}{ Average C/E } & $\begin{array}{c}\text { Average Experiment } \\
\text { Uncertainty } \\
(\mathrm{pcm})\end{array}$ & $\begin{array}{c}\text { St. Dev. from } \\
\text { C/E values } \\
(\mathrm{pcm})\end{array}$ & $\begin{array}{c}\text { St. Dev. from } \\
\text { ENDF/B-VII.1 } \\
\text { Cov }(\mathrm{pcm})\end{array}$ & $\begin{array}{c}\text { St. Dev. from } \\
\text { SCALE-6.2 } \\
\text { Cov }(\mathrm{pcm})\end{array}$ \\
\hline HST & 0.99624 & 0.99607 & 482 & 245 & 1100 & 812 \\
LST & 0.99847 & 0.99776 & 219 & 260 & 1139 & 556 \\
LCT & 0.99948 & 0.99893 & 318 & 185 & 921 & 597 \\
\hline \hline
\end{tabular}

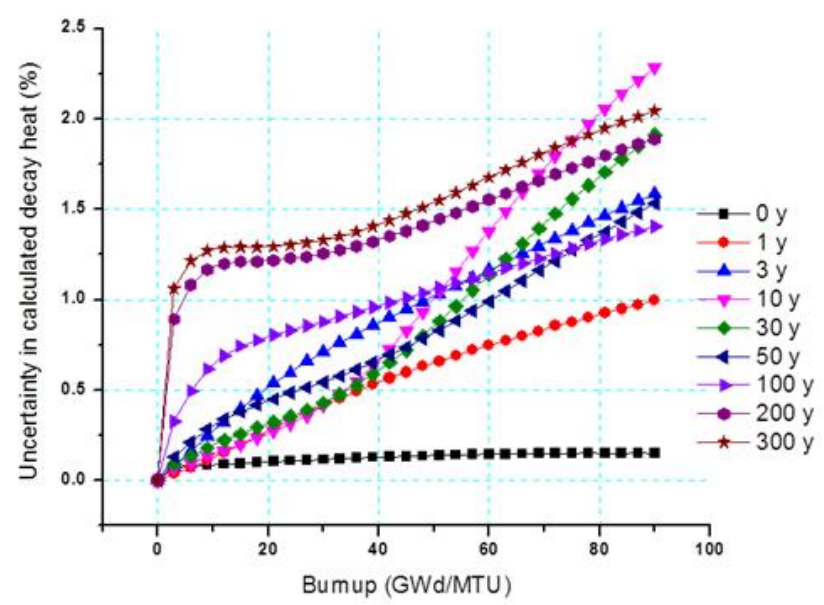

FIG. 5. (Color online) Uncertainties in UNF decay heat for various cooling times.

\section{SUMMARY}

Nuclear data covariances are now beginning to fulfill the long-anticipated goal of supporting uncertainty analysis for many types of applications. Previously, SCALE SU methods were based on computing sensitivity coefficients with generalized perturbation theory, which limited the applications to certain types of responses such as eigenvalues and reaction rate ratios. However, a new capability has been developed for SCALE-6.2 to compute response uncertainties by direct statistical sampling of nuclear data covariances, which has enlarged the classes of problems that can be addressed. The next release of the SCALE code system, version 6.2, will include cross section covariance data based on ENDF/B-VII.1 with a few modifications, as well as covariances for fission product yields and decay data.

Application of SCALE SU methods to a number of uranium thermal benchmarks suggests that currently available evaluated covariance data produce overly conservative (i.e., too high) values for the eigenvalue uncertainties. To obtain a more realistic estimate for the eigenvalue uncertainties in criticality safety reactor analysis, it may be necessary to apply a generalized least-squares adjustment to reduce the a priori uncertainty. The statistical sampling method was applied to the SCALE lattice physics/depletion sequence for the analysis of decay heat produced by a burned pressurized water reactor fuel assembly. The impact of nuclear data uncertainties taken from the SCALE-6.2 covariance library was propagated through all time steps of the burnup simulation - including self-shielding, nuclide transmutation, and space-energy flux transport calculations - to assess the uncertainty in decay heat as a function of time after shutdown. The decay heat uncertainty was found to vary with both exposure and decay time, with a maximum standard deviation of $2.5 \%$.

Acknowledgements: This work was sponsored by the US Department of Energy, Nuclear Criticality Safety Program.
[1] "SCALE: A Comprehensive Modeling and Simulation Suite for Nuclear Safety Analysis and Design," Oak Ridge National Laboratory Report ORNL/TM-2005/39, Version 6.1 (2011).

[2] M.L. Williams et al., "A Statistical Sampling Method for Uncertainty Analysis with SCALE and XSUSA," NuCL. TEChNOL. 183, 515-527 (2013).

[3] B.T. Rearden et al., "Sensitivity and Uncertainty Analysis Capabilities in SCALE," NuCl. TeChnol. 174, 236288 (2011).

[4] M.E. Dunn and N.M. Greene, "AMPX-2000: A CrossSection Processing System for Generating Nuclear Data for Criticality Safety Applications," Trans. Am. Nucl. Soc. 86, 118-119 (2002).
[5] M.B. Chadwick et al., "ENDF/B-VII.1 Nuclear Data for Science and Technology: Cross Sections, Covariances, Fission Product Yields and Decay Data," Nucl. DATA SheEts 112, 2887-2996 (2011).

[6] M.L. Williams and B.T. Rearden, "Sensitivity/Uncertainty Analysis Capabilities and New Covariance Data Libraries in SCALE," NuCl. DATA SHEETs 109, 2796-2800 (2008).

[7] Some concern was expressed about reduction of the ${ }^{235} \mathrm{U}$ total nubar thermal uncertainty in SCALE-6.2 from $0.7 \%$ to $3 \%$. The ENDF/B-VII. $1{ }^{235} \mathrm{U}$ nubar evaluation was performed by Los Alamos. It appears that the prompt (MT-456) and delayed (MT-455) are reasonable, but the total nubar (MT-452) is about a factor of two too high. 
Our uncertainty analysis only uses total nubar, while most Monte Carlo codes use the prompt and delayed components individually. This is probably why the inconsistency was not found earlier.

[8] M.T. Pigni, M.W. Francis and I.C. Gauld, "Investigation of Inconsistent ENDF/B-VII.1 Independent and Cumulative Fission Product Yields with Proposed Revisions," NuCl. Data Sheets 123, 231 (2015).

[9] "International Handbook of Evaluated Criticality Safety Benchmark Experiments," Nuclear Energy Agency Re- port NEA/NSC/DOC(95)03, Paris (2005).

[10] M.D. DeHart and S.M. Bowman, "Reactor Physics Methods and Analysis Capabilities in SCALE," NuCL. TECHNOL. 174, 196-213 (2011).

[11] M.L. Williams, G. Ilas, W.J. Marshall, and B.T. Rearden, "Applications of Nuclear Data Covariances to Criticality for Safety and Spent Fuel Characterization," NuCl. DATA SHEETs 118, 341 (2014). 